\title{
Imaging Review of Adolescent Tibial Tuberosity Fractures
}

\author{
Pranav Chitkara, Raja Anne, Sherlin Lavianlivi, Scott Lehto, Srinivas Kolla \\ Department of Radiology, SUNY Downstate Medical Center, Brooklyn, USA \\ Email: Pranav.Chitkara@downstate.edu
}

Received July 9, 2013; revised August 6, 2013; accepted August 20, 2013

Copyright (C 2013 Pranav Chitkara et al. This is an open access article distributed under the Creative Commons Attribution License, which permits unrestricted use, distribution, and reproduction in any medium, provided the original work is properly cited.

\begin{abstract}
Adolescent tibial tuberosity injuries are infrequent fractures usually seen in physically active adolescent males. Powerful contraction of the knee extensors by sudden acceleration or deceleration of the quadriceps muscle can result in avulsion fractures of the tibial tuberosity apophysis. In late puberty, as the growth plate closes, it is transiently replaced by fibrocartilaginous elements. This transition causes a period of weakened tensile strength, which predisposes the tibial tuberosity to traction injury. Classification of tibial tuberosity fractures includes types I-V with added A and B subsets to types I, II and III. Multidetector computed tomography (MDCT) is a useful tool to more accurately classify complex, higher grade adolescent tibial tuberosity avulsion fractures when compared to plain film. This aids in preoperative planning and, therefore, results in improved treatment and management.
\end{abstract}

Keywords: Tibial Tuberosity; Avulsion; Fracture; Radiology; Orthopedic; MDCT

\section{Introduction}

Tibial tuberosity avulsion injuries are infrequent fractures with a reported incidence ranging from $0.4 \%$ to $2.7 \%$. They represent approximately $3 \%$ of all proximal tibial fractures [1,2]. These avulsion injuries predominantly occur in well-developed athletic males as the tibial physis begins to fuse before skeletal maturity, which occurs near $14-17$ years of age.

It is suspected that female patients develop this injury less frequently than males because females undergo physiodesis of the proximal tibial tubercle at a younger age [1]. In addition, a greater percentage of males participate in athletics during adolescence and therefore subject their tibial tubercle to greater stress during their respective period of physiodesis [3]. In the majority of cases, tibial tubercle avulsion fractures are sustained through jumping activities. Several sports, including running, gymnastics, springboard diving, and football have been implicated with this injury. However, high jumping and basketball are most often associated $[1,3,4]$.

\section{Mechanism}

At skeletal maturity, the tibial tubercle is a prominent bony structure approximately $3 \mathrm{~cm}$ distal to the proximal articular surface of the tibia. It serves as the attachment for the quadriceps muscle via the patellar tendon. The tibial apophysis is vulnerable before and during physiol- ogic physiodesis. If an action causes the patellar ligament to create a force that exceeds the combined strength of the physis and surrounding perichondrium and periosteum, an avulsion fracture can occur [1]. Two mechanisms have been described to illustrate this injury.

The first mechanism involves knee extension during strong quadriceps contraction [1]. This can occur during the take off from a jump resulting in violent quadriceps contraction against a fixed leg without shortening [5]. The second mechanism is represented by landing from a jump or after falling from a height. This involves rapid passive flexion of the knee against a contracting quadriceps [1].

Osgood-Schlatter disease also involves the tibial tuberosity. However, it differs from tubercle fractures as there is only avulsion of the anterior portion of the tubercle and the physis is not involved. Past articles and case reports have suggested that Osgood-Schlatter disease is a possible predisposing factor to acute avulsion of the entire tuberosity $[6,7]$. However, recent articles contend that these prior cases did not effectively demonstrate a direct cause and effect relationship between the two entities [1].

\section{Pathophysiology}

The tibial tuberosity develops from a secondary ossification center in the proximal tibia. It is an apophysis that develops under traction in contrast to the proximal tibial 
epiphysis, which develops in compression [8].

It is important to understand the four stages of tubercle development. Cartilaginous, apophyseal, epiphyseal, and bony stages (Table 1) have been described by Ehrenborg et al. [9]. The cartilaginous and apophyseal stages are separated by the appearance of the secondary ossification center. Next, the epiphyseal stage occurs when the ossification centers of the proximal tibial epiphysis and tubercle join. This results in continuity of the tubercule with the proximal tibial epiphysis. Last, the bony stage is distinguished by bony fusion between the ossified tuberosity and the proximal tibial metaphysis. The bony stage occurs in girls by age 15 and in boys by age 17 [1,9].

In addition to these stages, there are histologic zones within the apophysis that influence development of avulsion fractures [1]. Three types of tubercle histology were noted by Ogden et al. The proximal portion of the tubercle consists of columnar cartilage, while the middle portion of the tubercule is composed of fibrocartilage. Moving distally along the tubercle, the fibrocartilage transforms into fibrous tissue, which that then blends with the perichondrium [10].

During skeletal maturation, fibrocartilage in the midportion transforms into columnar cartilage around the time of physiologic physiodesis. Physeal fusion then begins in the center of the proximal tibial physis and progresses centrifugally and distally along the tubercle [1]. While fibrocartilage is resistant to tensile stress, columnar cartilage is weak when subjected to such stress [6].

Maturation of the tibial tubercle results from this combination of osseous and apophyseal changes. Just before or during physiologic physiodesis, an interval of vulnerability is created predisposing the tuberosity to avulsive injury [1].

\section{Classification}

Classification of tibial tubercle fractures has evolved since they were first described in 1976. Initially, the Watson-Jones classification detailed types I, II and III. Then, Ogden modified the classification to better define the extent of injury and amount of displacement or comminution by adding A and B subsets to those types [2]. Type IV was added by Ryu and Debenham to describe an

Table 1. Stages of tubercle development [1,9].

\begin{tabular}{ccc}
\hline Stage & Girls (age) & Boys (age) \\
\hline Cartilaginous & $<8$ & $<9$ \\
Apophyseal & $8-12$ & $9-14$ \\
Epiphyseal & $10-15$ & $11-17$ \\
Bony & 15 & 17 \\
\hline
\end{tabular}

avulsion fracture of the entire proximal tibial epiphysis [11]. Subsequently, type V was introduced to the classification system by McKoy et al. to describe their experience with a patient exhibiting both a type IIIb and type IV injury [1]. The addition of subset $C$ was then suggested for type I fractures with associated patellar tendon tears by Frankl et al. [12].

\subsection{Type I}

Type I tibial tuberosity fractures exhibit injury of the distal portion of the apophysis. The fracture line is present through the tubercle ossification center between the proximal tibia and tuberosity. Type IA indicates a minimally displaced or non-displaced fracture (Figure 1), while type IB (Figure 2) signifies anterior and proximal displacement or comminution [6,13]. A type IC designation was proposed to describe associated patellar tendon avulsions [12].
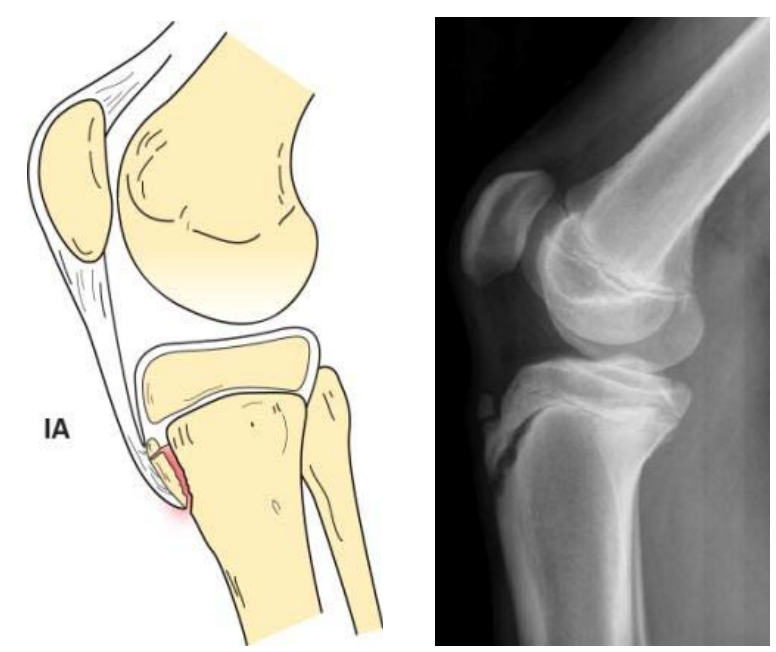

Figure 1. Type IA schematic and radiographic representation.

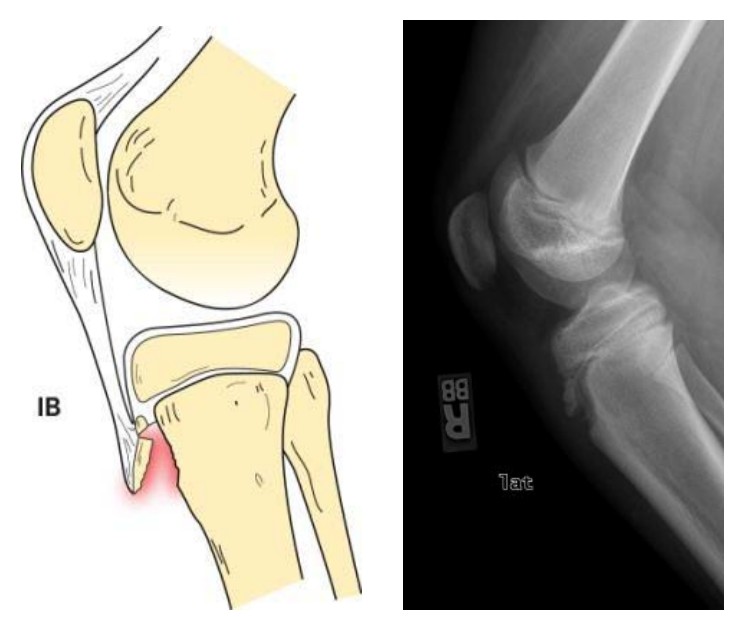

Figure 2. Type IB schematic and radiographic representation. 


\subsection{Type II}

The Type II classification is manifest by proximal fracture extension through the cartilage between the proximal tibial epiphysis and tubercle without articular involvement. If the fracture is minimally or non-displaced without comminution, it is designated as type IIA (Figure 3). If there is anterior displacement and/or comminution of the fracture (Figure 4), it is described as type IIB [1,2, $6,13]$.

\subsection{Type III}

Type III tibial tuberosity fractures demonstrate anterior intra-articular fracture extension through the physis and epiphysis into the knee (Figure 5). The tuberosity and the anterior epiphysis remain as a unit. A type IIIB designation (Figure 6) is given if the avulsion results in comminution $[1,2,6]$.
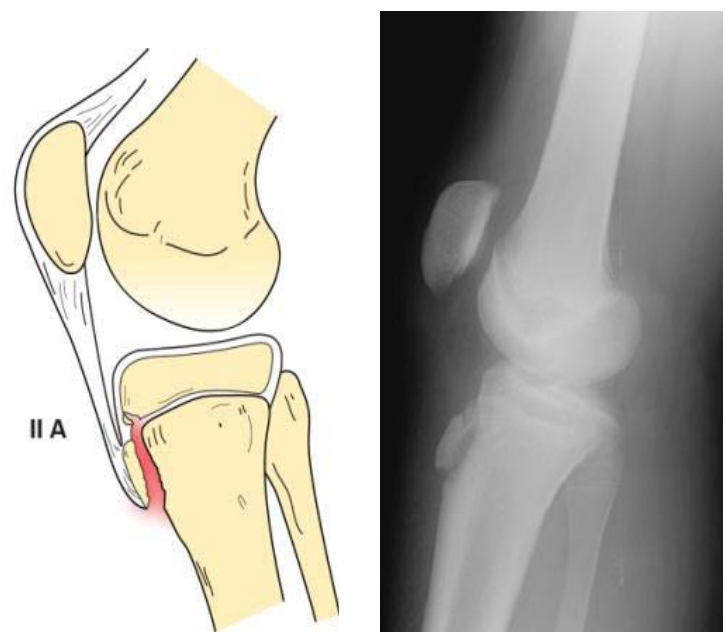

Figure 3. Type IIA schematic and radiographic representation.
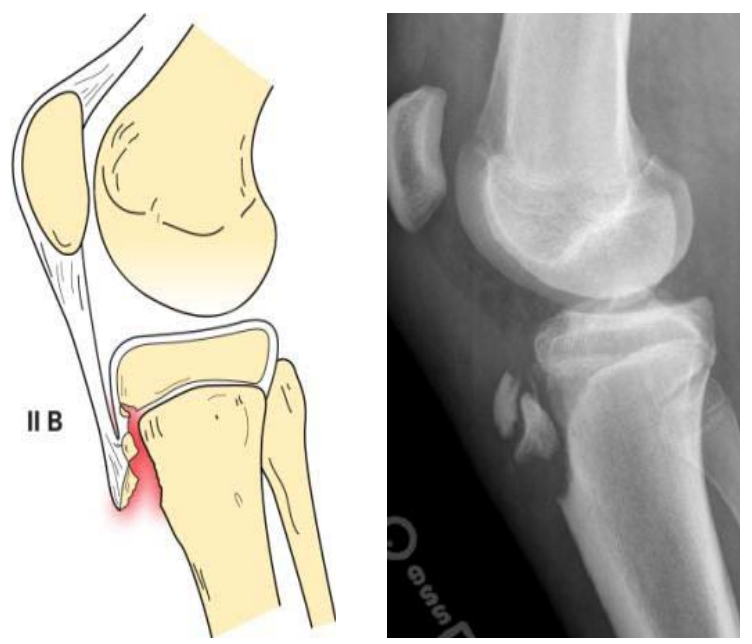

Figure 4. Type IIB schematic and radiographic representation.

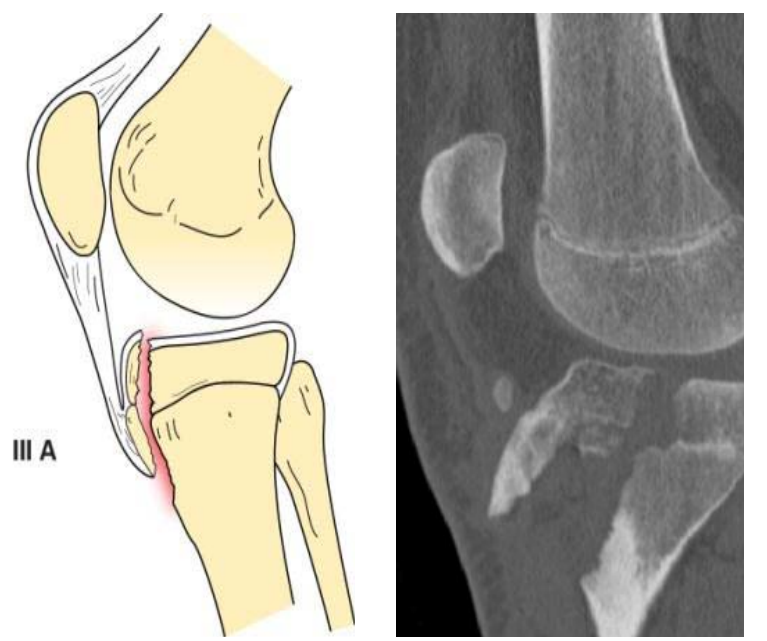

Figure 5. Type IIIA schematic and radiographic representaion.
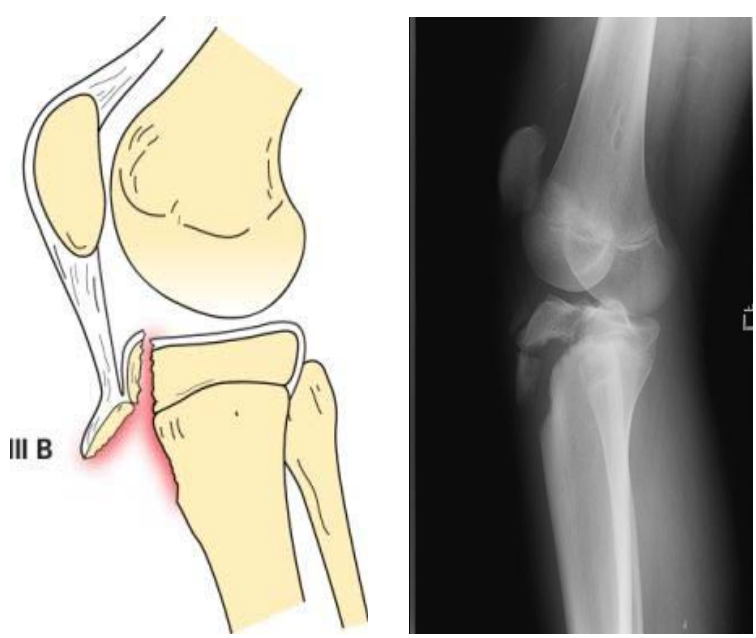

Figure 6. Type IIIB schematic and radiographic representation.

\subsection{Type IV}

Type IV tibial tuberosity avulsion presents with fracture extension from the tibial tuberosity, posteriorly through the proximal tibial physis, and then into the posterior tibial metaphyseal cortex (Figure 7). These fractures will present with entire proximal tibial physeal separation [1, $11,14]$.

This fracture type has a low incidence because the proximal tibial epiphysis does not normally serve as an insertion point for the medial or lateral collateral ligaments. Therefore, the varus and valgus stresses are transmitted to the metaphysis rather than to the epiphysis [14].

\subsection{Type V}

Type V tibial tuberosity avulsion consists of a combined type III and type IV avulsion fracture (Figure 8). This creates an avulsion fracture with articular involvement 


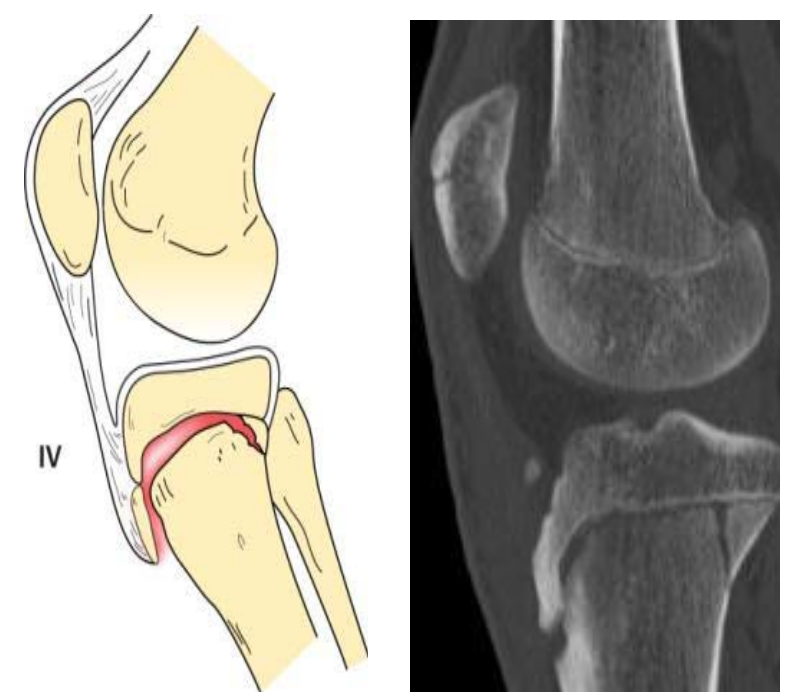

Figure 7. Type IV schematic and radiographic representation.
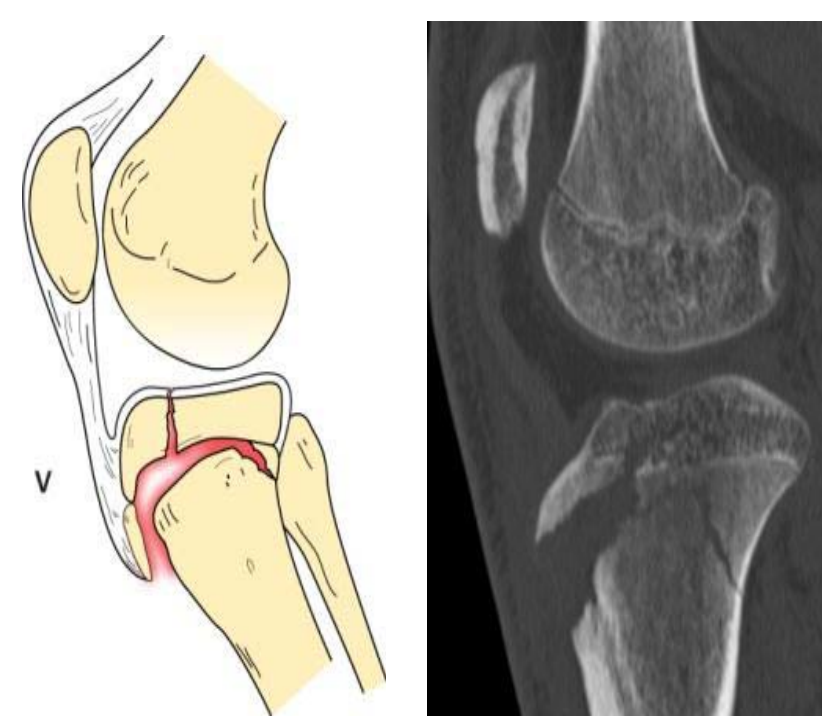

Figure 8. Type V schematic and radiographic representation.

through the physis and epiphysis. In addition, there is complete separation of the proximal tibia through physeal extension posteriorly. This results in an inverted "Y" configuration $[1,2]$.

\section{Clinical Presentation}

Patients with tibial tuberosity fractures commonly experience swelling, pain, and tenderness directly over the tuberosity. In type I injuries, knee extension against gravity is preserved, however extension against resistance is compromised. In type II injuries and greater, extension against gravity with and without resistance is limited. In addition, knee extension in the decubitus position with gravity is also compromised [6].

\section{Imaging}

The initial imaging study for tibial tubercle fractures is plain radiography. AP and lateral views are essential to make the diagnosis, but the lateral view best depicts the size and displacement of the fragment [1]. Oblique radiographs of the proximal tibia can be useful to better demonstrate the tubercle as it lies just lateral to the midline [15].

Although standard radiographs are helpful in diagnosing the complex fracture pattern, precise configuration is only established by computed tomography. Advanced imaging can result in upgrading the classification of some avulsion injuries compared to initial evaluation with plain film. CT imaging and 3D volume rendering (Figures 9-12) are useful to these recognize complex fracture patterns and aid in preoperative planning [16].

MR imaging can be performed to evaluate for meniscal and ligament injury or cartilage damage.

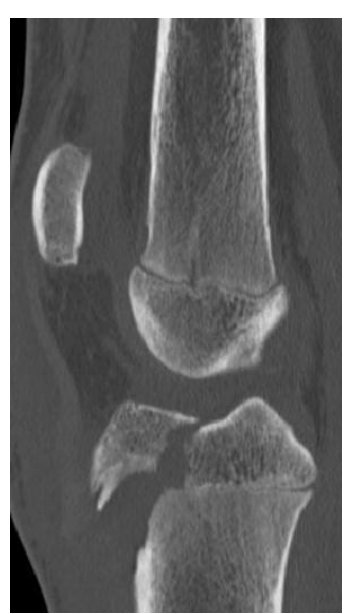

(a)

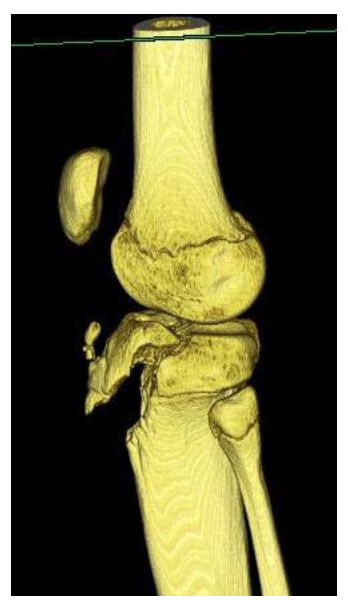

(c)

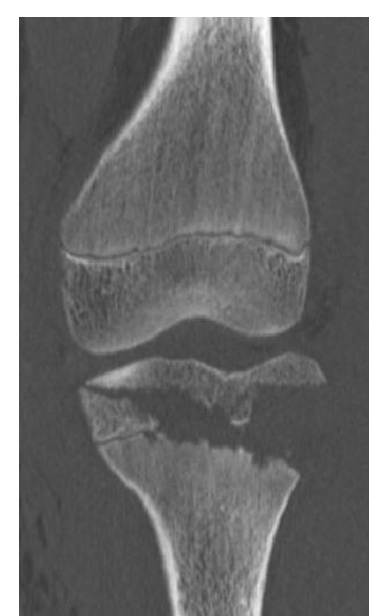

(b)

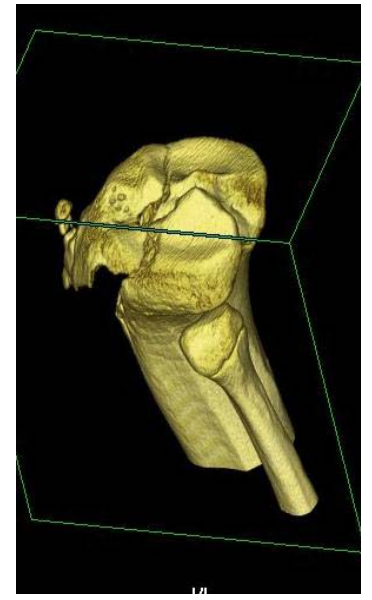

(d)
Figure 9. 13-year-old male with a type IIIA fracture. MDCT reformations (a) and (b) and 3-D volume rendered images (c) and (d) demonstrating avulsion of the anterior “apophysis-epiphysis” unit with articular involvement. 

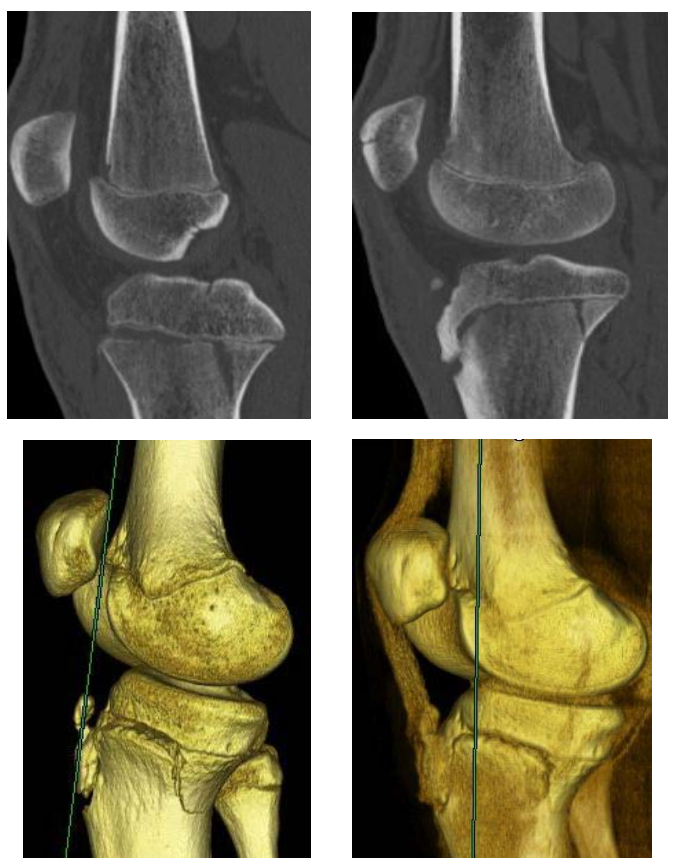

Figure 10. 15-year-old male with a type IV fracture. MDCT reformations and 3-D volume rendered images demonstrating avulsion of the tibial tuberosity with fracture extension posteriorly along the epiphysis and with involvement of posterior metaphysis.
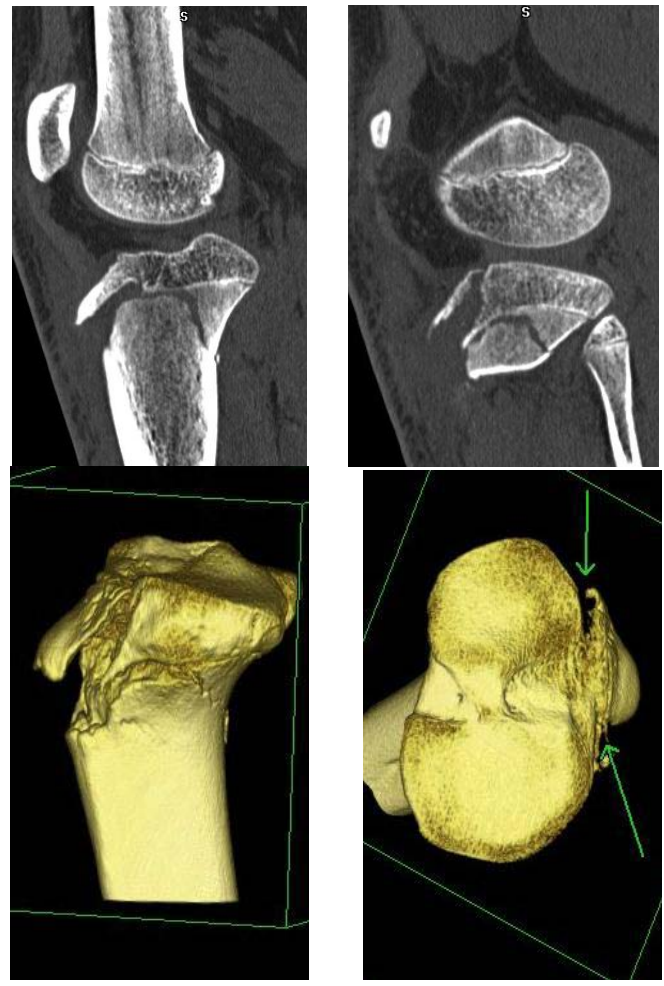

Figure 11. 15-year-old male with a type V fracture. MDCT reformations and 3-D volume rendered images demonstrating avulsion of the tibial tuberosity with intra-articular extension (arrows) and fracture extension posteriorly along the epiphysis and posterior metaphysis.

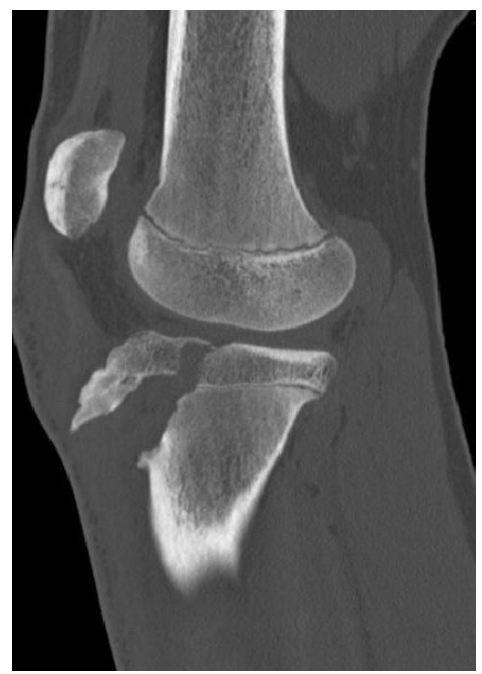

Figure 12. MDCT sagittal reformation demonstrating a type IIIA fracture.

\section{Management}

For type IA and IB fractures, treatment is conservative with closed reduction and casting as long as the extensor mechanism remains intact [17]. For type IC, however, open reduction internal fixation (ORIF) of the avulsed osseous fragment and ligament is recommended [12].

Chow et al. described successful treatment of 10 patients with type IA and IIA injuries with closed reduction plaster casting for approximately six weeks. However, one patient required surgical repair for a ruptured patellar tendon [17]. Ogden et al. treated six children with type I injuries by cylinder cast immobilization with the knee in complete extension for three to six weeks. In one patient, the fragment had separated completely (a type IB lesion) requiring open reduction and fixation with a screw into the metaphysis [6].

Open reduction and internal fixation is performed for types IIB, IIIA, IIIB, and IV tibial tuberosity avulsion fractures [1,3,4,6,17]. In addition, associated quadriceps or patellar tendon avulsions should be repaired to restore the extensor mechanism [1]. Several methods of fixation have been reported, but most authors recommend tension band wiring or cannulated screw fixation of the avulsed fragment (Figure 13) [1,13]. Arthroscopy can also be considered if there is concern for concomitant internal derangement [2]. Despite the method of surgical intervention, the aim is anatomic reduction of fragment, restoration of extensor mechanism alignment, and maintenance of the tibial articular surface [17].

In general, less severe classifications of avulsion fracture of the tibial tuberosity can be treated conservatively (Figure 14). When surgical intervention is necessary, a cancellous screw and tension band wiring are the treatment of choice (Table 2) [4,7]. 


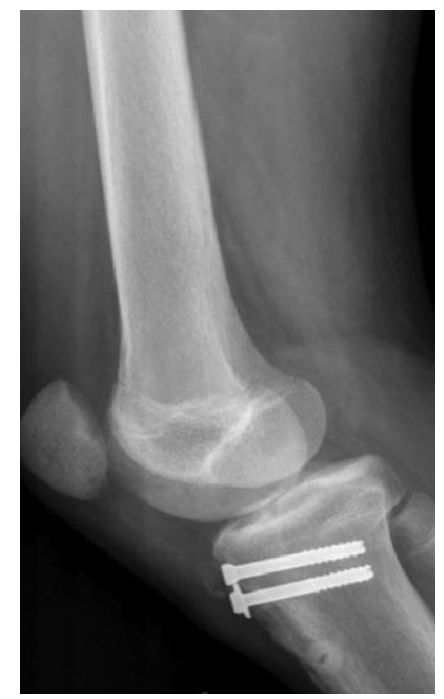

Figure 13. Type IIIA fracture post internal fixation.

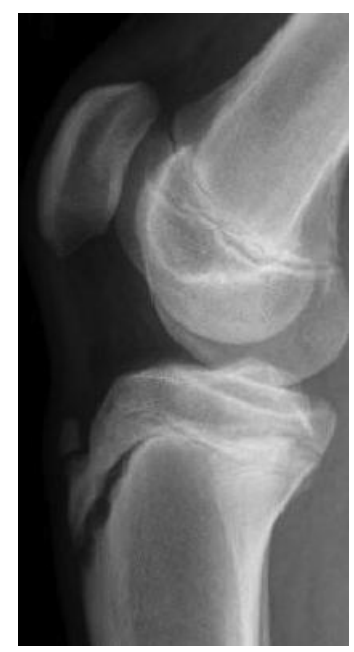

(a)

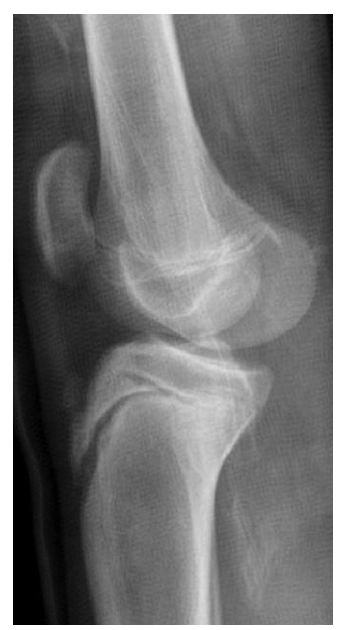

(b)

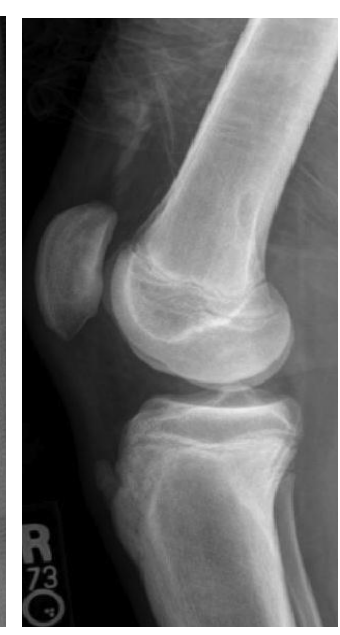

(c)
Figure 14. A 17-year-old male with Type IA fracture (a) treated with long leg cast (b) with eventual complete healing (c).
Table 2. Imaging and management.

\begin{tabular}{ccc}
\hline Type & Imaging & Management \\
\hline IA/B, IIA & Plain Radiography & $\begin{array}{c}\text { Closed } \\
\text { reduction and casting. } \\
\text { IIB, IIIA/B, IV , \& V }\end{array}$ \\
MDCT & $\begin{array}{c}\text { Open reduction and } \\
\text { internal fixation. }\end{array}$ \\
\hline
\end{tabular}

\section{Discussion}

Adolescent tibial tuberosity fractures are uncommon with a reported incidence of $0.4 \%$ to $2.7 \%$ [1,2]. The two mechanisms of injury are 1) quadriceps contraction against a fixed leg without shortening and 2) forceful flexion of the knee against contraction of the quadriceps. These injuries are most often seen in physically active adolescent males and are associated with sports involving jumping activities. Common activities causing injury include basketball, high jumping, volleyball, sprinting, and falling from a height.

The tibial tuberosity forms from a secondary ossification center in the proximal tibia that develops under traction. During closure of the proximal tibial physis, a mechanically vulnerable period is created, which predisposes the tuberosity to avulsion injury. With skeletal maturation, the fibrocartilage originally in the mid-portion of the tubercle transforms into columnar cartilage. While fibrocartilage is resistant to tensile stress, columnar cartilage is weak when subjected to such stress [6]. During physiodesis, if an action causes the patellar tendon to create a force that exceeds the combined strength of the physis and surrounding perichondrium and periosteum, then an avulsion fracture will occur.

There has been an evolution of the tibial tubercle fracture classification since types I, II, and III were first introduced by Watson-Jones. Subsequently, Ogden, Ryu, McKoy, and several others have made contributions. Currently, there are 5 types with an A and B subclassification of types I, II, and III.

Regarding treatment, type IA/B and IIA fractures are treated conservatively with closed reduction as long as the extensor mechanism is intact. Classification IIB injuries and above are approached with open reduction-internal fixation. In general, outcomes are excellent with complete restoration of the extensor mechanism.

The initial modality for imaging of tibial tuberosity injuries has traditionally been plain films. In some cases, however, plain films can underestimate the degree of injury. Advanced imaging is then advocated to evaluate higher grade injuries and assist the orthopedic surgeon with preoperative planning and precise evaluation of the fracture classification.

\section{REFERENCES}

[1] B. E. McKoy and C. L. Stanitski, “Acute Tibial Tubercle 
Avulsion Fractures," Orthopedic Clinics of North America, Vol. 34, No. 3, 2003, pp. 397-403. doi:10.1016/S0030-5898(02)00061-5

[2] S. Frey, et al., "Tibial Tuberosity Fractures in Adolescents,” Journal of Children's Orthopaedics, Vol. 2, No. 6, 2008, pp. 469-474. doi:10.1007/s11832-008-0131-z

[3] S. M. Mosier, C. L. Stanitski and R. S. Levine, "Simultaneous Bilateral Tibial Tubercle Avulsion Fracture,” Orthopedics, Vol. 23, No. 10, 2000, pp. 1106-1108.

[4] M. J. Bolesta and R. D. Fitch, "Tibial Tubercle Avulsions,” Journal of Pediatric Orthopaedics, Vol. 6, No. 2, 1986, pp. 186-192. doi:10.1097/01241398-198603000-00013

[5] R. Watson-Jones, "Fractures and Joint Injuries," 4th Edition, Williams \& Wilkins, Baltimore, 1955.

[6] J. A. Ogden, et al., "Fractures of the Tibial Tuberosity in Adolescents," Journal of Bone and Joint Surgery (American Volume), Vol. 62, No. 2, 1980, pp. 205-215.

[7] S. A. Buhari, S. Singh, et al., "Tibial Tuberosity Fractures in Adolescents,” Singapore Medical Journal, Vol. 34, No. 5, 1993, pp. 421-424.

[8] J. A. Ogden, W. O. Southwick, “Osgood-Schlatter's Disease and Tibial Tuberosity Development," Clinical Orthopaedics and Related Research, No. 116, 1976, pp. 180-189.

[9] G. Ehrenborg, B. Engfeldt, "The Insertion of the Ligamentum Patellae on the Tibial Tuberosity. Some Views in Connection with the Osgood-Schlatter Lesion,” Acta chirurgica Scandinavica, Vol. 121, 1961, pp. 491-499.

[10] J. A. Ogden, R. J. Hempton and W. O. Southwick, "Development of the Tibial Tuberosity," The Anatomical Re- cord, Vol. 182, No. 4, 1975, pp. 431-445. doi:10.1002/ar.1091820404

[11] R. K. Ryu and J. O. Debenham, “An Unusual Avulsion Fracture of the Proximal Tibial Epiphysis. Case Report and Proposed Addition to the Watson-Jones Classification," Clinical Orthopaedics and Related Research, No. 194, 1980, pp. 181-184.

[12] U. Frankl, S. A. Wasilewski and W. L. Healy, "Avulsion Fracture of the Tibial Tubercle with Avulsion of the Patellar Ligament. Report of Two Cases,” Journal of Bone and Joint Surgery (American Volume), Vol. 72, No. 9, 1990, pp. 1411-1413.

[13] T. Pesl and P. Havranek, "Acute Tibial Tubercle Avulsion Fractures in Children: Selective Use of the Closed Reduction and Internal Fixation Method," Journal of Children's Orthopaedics, Vol. 2, No. 6, 2008, pp. 469474.

[14] B. K. K. Fung and Y. H. Li, "Avulsion Fracture of the Tibial Tubercle and Proximal Tibialphysis," Hong Kong Journal of Orthopaedic Surgery, Vol. 5, No. 1, 2001, pp. 70-73.

[15] C. S. Dupuis, S. J. Westra, J. Makris and E. C. Wallace, "Injuries and Conditions of the Extensor-Mechanism of the Pediatric Knee,” Radiographics, Vol. 29, 2009, pp. 877-886. doi:10.1148/rg.293085163

[16] A. Jalgaonkar, S. Dachepalli, et al., "Atypical Tibial Tuberosity Fracture in an Adolescent," Orthopedics, Vol. 34, No. 6, 2011, p. 215.

[17] S. P. Chow, et al., "Fracture of the Tibial Tubercle in the Adolescent," The Bone \& Joint Journal (British Volume), Vol. 72, No. 2, 1990, pp. 231-234. 